\title{
Porcine pancreas mesenchymal cell characterization and functional differentiation into insulin-producing cells in vitro
}

\author{
SHANG ZHANG, QI WANG, HONGBING JI, HUIDI LU, QIN YANG, JIAHUI YIN and WEIJUN GUAN \\ Department of Animal Genetic Resources, Chinese Academy of Agricultural Sciences, Beijing 100193, P.R. China
}

Received June 23, 2020; Accepted January 5, 2021

DOI: $10.3892 / \mathrm{mmr} .2021 .12377$

\begin{abstract}
Cell therapy is a promising treatment strategy for patients with type 1 diabetes. Porcine pancreas-derived mesenchymal stromal cells (PMSCs) have emerged as one of the most widely used cell resources owing to their high proliferative capacity and multi-lineage differentiation potential. Although the induction efficiency and insulin production of induced insulin-producing cells (IPCs) derived from PMSCs have been estimated, these have primarily focused on the function of induced cells and alterations in related gene expression levels. However, morphological analyses and biological characterization of PMSCs and induced IPCs have not been conducted. Therefore, the present study aimed to optimize an induction protocol, resulting in a $78.92 \%$ induction rate. The present study investigated the biological characteristics of PMSCs and optimized a simple but functional three-step protocol to transform PMSCs into IPCs. PMSCs were isolated from 2-3-month-old Bama miniature pig embryos, which were then subcultured to passage 16 . The surface markers pancreatic and duodenal homeobox 1, NK6 homeobox 1, Vimentin, Nestin, CD73, CD90, neurogenin 3, CD45 and CD34 were detected by immunofluorescence staining or flow cytometry. Proliferative capacity was evaluated by constructing growth curves of cells at three different passages. Functional differentiation was assessed by morphological observation, dithizone staining, and immunofluorescence staining of C-peptide, insulin, NK6 homeobox 1 and glucagon. The production of insulin by differentiated cells was also analyzed by performing ELISAs. The results demonstrated that differentiated cells were distributed with an islet-like structure, expressed specific markers C-peptide and insulin, and displayed glucose responsiveness. The results of the present study demonstrated that PMSCs were functionally induced into IPCs with the optimized three-step
\end{abstract}

Correspondence to: Professor Weijun Guan, Department of Animal Genetic Resources, Chinese Academy of Agricultural Sciences, 2 Yuanmingyuan Xilu Road, Haidian, Beijing 100193, P.R. China

E-mail: weijunguan@caas.cn

Key words: pancreatic mesenchymal stem cells, insulin-producing cells, Bama miniature pigs, diabetes, cell therapy protocol, which may serve as a potential cell therapy strategy to widen the availability and promote the clinical application of cell therapy.

\section{Introduction}

Diabetes is a global epidemic that affects 300 million individuals worldwide and is one of the primary threats to human health, with a markedly increasing incidence (1). Hyperglycemia is a feature of diabetes mellitus and can cause numerous complications, including heart disease, cataracts, kidney disease and nerve damage $(1,2)$. The primary causes of all forms of diabetes include the loss of insulin or insufficient quantities of insulin production/secretion from pancreatic $\beta$-cells in the islets of Langerhans (3).

The pancreas is the second largest gland in the body and is composed of endocrine and exocrine components (3). Exocrine islets form an important digestive system that produces digestive enzymes, including chymotrypsin, amylase and lipase, which are used to digest proteins, and degrade carbohydrates and fats. Endocrine islets are the source of several hormones, including insulin and glucagon (4,5). Insulin, which is secreted by $\beta$ cells in the pancreas, is the most important metabolic hormone for controlling blood glucose. Loss of islet $\beta$ cells may lead to diabetes and require therapeutic intervention.

Regenerative medicine is considered as a valuable therapeutic approach that has gained increasing acceptance worldwide. Devising potential strategies to develop insulin-producing $\beta$-cells might result in profound effects for the treatment of diabetes. Previous studies in fish (6), mice (7) and frogs (8) have demonstrated the differentiation potential of multipotent stem cells (SCs) (5). Controlled differentiation of SCs, including embryonic (9), pancreatic (10) and bone marrow mesenchymal (11) SCs, into functional insulin-producing cells (IPCs) fulfills the requirement of transplantable $\beta$ cells. However, the protocol for the controlled differentiation of SCs is not completely understood.

Pancreas-derived mesenchymal stromal cells (PMSCs) are one of the types of mesenchymal cells isolated from pancreatic tissues. Compared with other cells types, PMSCs are highly enriched during vascular development, chemotaxis and wound healing (12). PMSCs also possess the ability to decrease hyperglycemia in type 1 diabetes model mice after intrapancreatic injection (12). PMSCs are a potential therapeutic agent for regenerative medicine applications (13). Recently, 
Lee et al (13) reported a two-step induced protocol to transform porcine-derived mesenchymal cells into insulin-producing cells; although the function of induced cells was estimated, the biological characterization and morphological alterations of the induced cells were not described.

In the present study, Bama miniature pig-derived mesenchymal stem cells (MSCs) were selected as the experimental cell source for a number of reasons: i) Swine are an appropriate animal model for humans, as they possess a high degree of similarity in tissue structure and genetics with humans (14); ii) MSCs with high proliferative capacity can be transformed into various cell types and display a vital role in regenerative medicine (15); and iii) SCs derived from the pancreas are more easily differentiated into functional $\beta$ cells compared with other cells (3). The present study described a novel protocol for generating pancreatic islet $\beta$ cells that may serve as a model to facilitate the study of the mechanisms controlling islet cell neogenesis and may aid in the identification of pharmaceutical targets to treat insulin-dependent diabetes.

\section{Materials and methods}

PMSC isolation, culture and purification. All animal procedures were approved by the Institutional Animal Care and Use Committee of Chinese Academy of Agricultural Sciences (approval no. IAS 2018-19, Beijing, China). The 2-3-month-old Wuzhishan miniature pig (Sus scrofa; also known as Bama miniature pigs) was provided by the Animal Husbandry Experimental Base, Institute of Animal Sciences, Chinese Academy of Agricultural Sciences (16). The animals were sacrificed through overdose of ketamine $(100 \mathrm{mg} / \mathrm{kg}$; cat. no. 087K1253; Sigma-Aldrich; Merck KGaA) and xylazine (25 mg/kg; cat, no. KH070901; Hengrui Co.) (16). The pancreas was removed from each miniature pig embryo $(n=3)$. After thoroughly washing with PBS buffer, tissue sections were dissected into $1-\mathrm{mm}^{3}$ thick segments and isolated via collagenase digestion under sterile conditions. Tissues were digested with $5.5 \mathrm{mg} / \mathrm{ml}$ collagenase $\mathrm{P}$ (Roche Diagnostics) for $60 \mathrm{~min}$ at $37^{\circ} \mathrm{C}$ with gentle agitation every $10 \mathrm{~min}$. Following digestion, cells were neutralized with RPMI-1640 (Gibco; Thermo Fisher Scientific, Inc.) supplemented with 10\% FBS (Gibco; Thermo Fisher Scientific, Inc.). The cell suspension was filtered through a 74- $\mu \mathrm{m}$ mesh sieve and then centrifuged at $225 \mathrm{x} \mathrm{g}$ for $15 \mathrm{~min}$ at room temperature. Cell pellets were resuspended with RPMI-1640 supplemented with 10\% FBS and $1 \%$ penicillin-streptomycin (Gibco; Thermo Fisher Scientific, Inc.). Cells $\left(1.5 \times 10^{6}\right)$ were seeded into plates and incubated at $37^{\circ} \mathrm{C}$ with $5 \% \mathrm{CO}_{2}$. Following incubation for 3-4 h, $10^{4}$ non-adherent cells were removed and reseeded in a new plate. Cells were incubated with RPMI-1640 medium supplemented with $20 \mathrm{ng} / \mathrm{ml}$ human-derived fibroblast growth factor (bFGF; PeproTech, Inc.) and $20 \mathrm{ng} / \mathrm{ml}$ human-derived epidermal growth factor (EGF; PeproTech, Inc.) at $37^{\circ} \mathrm{C}$. After 12 h, 100s of cells started growing with a spindle shape. When the cells reached $80-90 \%$ adherence, they were digested with $0.125 \%$ trypsin and considered as passage 1 . Passage 4 , passage 13 and passage 18 cells were obtained by the same method. PMSCs were purified by the different adhesion time method (17). According to the preliminary experiment, PMSCs displayed a shorter adhesion time compared with other cells types, thus PMSCs could be purified after the third passage. In addition, PMSCs should be mesenchymal cell specific marker Vimentin positive and display fibroblast-like morphology; these were checked to assess the purity of isolated PMSCs. Contaminating cells, such as blood and duct cells, displayed a different adherence time and could be excluded by passaging.

Growth kinetics. Cells from passage 3, 8 and 16 were seeded $\left(1 \times 10^{4}\right.$ cell/well) into 24 -well plates. The cell suspension was absorbed and dropped onto the edge of the cell counting plate, and the mean value of four large squares was calculated. Cell counting was repeated three times to obtain a mean value for each large square. The cell count (cells/ml) was calculated as follows: The mean cell number $\times 10^{4}$. Cells were counted every day for 7 days. The population doubling time (PDT) was calculated according to the following formula: $\mathrm{PDT}=\left(\mathrm{t}-\mathrm{t}_{0}\right)$ $\log _{2} /\left(\log \mathrm{Nt}-\log \mathrm{N}_{0}\right)$, where $\mathrm{t}_{0}$ is the starting time, $\mathrm{t}$ is the termination time, $\mathrm{N}_{0}$ is the initial cell number and $\mathrm{Nt}$ is the ultimate cell number.

Colony-formation assay. Cells from passage 3, 8 and 14 were seeded $\left(1 \times 10^{4}\right.$ cell/well) into 24 -well plates and cultured for 4 days. Then, the cells were fixed with $4 \%$ paraformaldehyde for $30 \mathrm{~min}$ at room temperature and stained with GIMSA (cat. no. C0133, Beyotime Institute of Biotechnology) for $30 \mathrm{~min}$ at room temperature. Subsequently, the number of colony-forming units was counted to calculate the colony-forming rate $(\%)$ according to the following formula: (Number of colony-forming units/number of seeded cells) $\mathrm{x} 100$. The experiment was repeated three times to calculate the mean value. The light microscope was used for images at $\mathrm{x} 4$ magnification.

Immunofluorescence staining. Purified PMSCs were seeded into 6-well plates. At $70 \%$ confluence, cells were washed three times with PBS, fixed with $4 \%$ paraformaldehyde for $30 \mathrm{~min}$ at room temperature and permeabilized with $0.25 \%$ Triton X-100 (Sigma-Aldrich; Merck KGaA) for $20 \mathrm{~min}$ in room temperature. Following blocking with $10 \%$ normal goat serum (1:10; OriGene Technologies, Inc.) for $30 \mathrm{~min}$ at room temperature, cells were incubated for $1 \mathrm{~h}$ at $37^{\circ} \mathrm{C}$ in 3\% BSA (Sigma-Aldrich; Merck KGaA) supplemented with the following primary antibodies: Anti-pancreatic and duodenal homeobox 1 (Pdx1; 1:200; cat. no. ab47267; Abcam), anti-Vimentin (1:200; cat. no. ab8978; Abcam), anti-neurogenin 3 (Ngn3; 1:200; cat. no. bs-0922R; Beijing Biosynthesis Biotechnology Co., Ltd.), anti-Nestin (1:200; cat. no. ab221660; Abcam) and anti-CD45 (1:200; cat. no. ab10558; Abcam). Following washing three times with PBS, cells were incubated with a FITC-conjugated rabbit anti-goat secondary antibody (1:200; cat. no. ZF-0314; OriGene Technologies, Inc.) or anti-mouse Alexa Fluor secondary antibody (1:200; cat. no. sc-516608; Santa Cruz Biotechnology, Inc) at room temperature in the dark for $1 \mathrm{~h}$. Cells were washed three times in the dark and then incubated with $1 \mu \mathrm{g} / \mathrm{ml}$ DAPI (Sigma-Aldrich; Merck KGaA) for $15 \mathrm{~min}$ in room temperature. Stained cells were visualized and counted in eight randomly selected non-overlapping fields of view using a TE-2000-E inverted fluorescence microscope (Nikon Corporation). For the control group, PBS was used in place of primary antibodies. Samples were assessed in triplicate. 


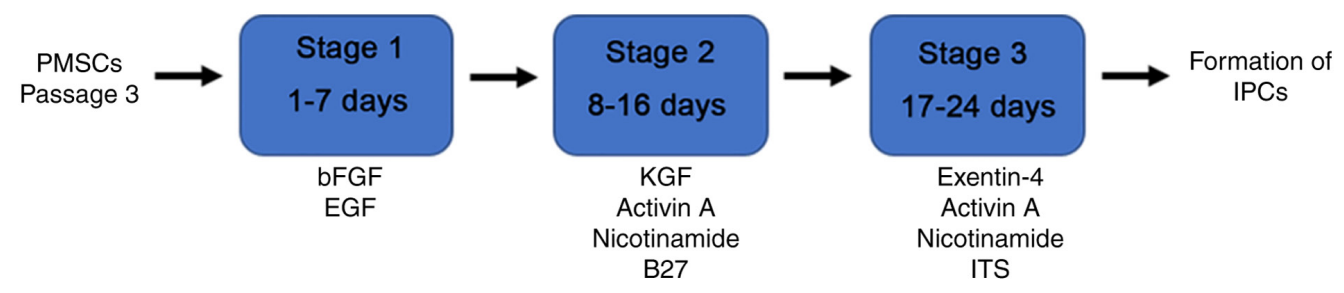

Figure 1. Generation of porcine-induced IPCs using an extended pluripotent stem cell culture system. IPCs were generated from PMSCs using a three-step protocol. bFGF, fibroblast growth factor; EGF, epidermal growth factor; ITS, insulin-transferrin-selenium; IPC, insulin-producing cell; KGF, keratinocyte growth factor; PMSC, pancreas-derived mesenchymal stromal cell.

Flow cytometry. Differentiated cell clusters or PMSCs both at a number of $10^{6}$ were dispersed into single-cell suspensions by incubation with trypsin for $1 \mathrm{~min}$ at $37^{\circ} \mathrm{C}$. Cells were fixed with $4 \%$ paraformaldehyde for $30 \mathrm{~min}$ at room temperature, washed with PBS and blocked with $1 \%$ normal goat serum for $20 \mathrm{~min}$ at room temperature. PMSCs were incubated with anti-CD73 (1:200; cat. no. ab175396; Abcam), anti-CD90 (1:200; cat. no. ab222781; Abcam) and anti-CD34 (1:200; cat. no. 81289; Abcam) primary antibodies for biological characterization at $4^{\circ} \mathrm{C}$ overnight. IPCs and PMSCs were resuspended in PBS with anti-insulin (1:200; cat. no. ab181547; Abcam) and incubated at $4^{\circ} \mathrm{C}$ overnight. Following washing twice with PBS, cells were incubated at room temperature with FITC-conjugated goat anti-rabbit IgG (1:200; cat. no. bs-40295G-HRP; BIOSS) and goat anti-mouse Alexa Fluor IgG (1:200; cat. no. bs-40296-HRP; BIOSS) secondary antibodies for $2 \mathrm{~h}$. For the control group, cells were only incubated with the secondary antibody and were not incubated with specific primary antibodies. Following washing, cells were analyzed using a Beckman Cytomics FC500 flow cytometer (Beckman Coulter, Inc.) and Flow software (Beckman CXP 2.1; Beckman Coulter, Inc.). Samples were assessed in triplicate. The assay was conducted in 60-mm dishes.

Transdifferentiation of PMSCs into IPCs in vitro. At passage 3, PMSCs were transdifferentiated into IPCs in vitro according to the protocol presented in Fig. 1. Firstly, PMSCs were incubated in medium supplemented with $20 \mathrm{ng} / \mathrm{ml}$ bFGF and $20 \mathrm{ng} / \mathrm{ml}$ EGF for 7 days. Subsequently, cells were transferred into medium containing $10 \mathrm{ng} / \mathrm{ml}$ keratinocyte growth factor (KGF; PeproTech, Inc.), $10 \mathrm{ng} / \mathrm{ml}$ Activin A (PeproTech, Inc.), $10 \mathrm{mmol} / 1$ nicotinamide (PeproTech, Inc.) and 2\% B27 supplement (PeproTech, Inc.) for 9 days. Cells were transferred into medium supplemented with $10 \mathrm{ng} / \mathrm{ml}$ Exendin-4 (PeproTech, Inc.), $10 \mathrm{ng} / \mathrm{ml}$ Activin A, $10 \mathrm{mmol} / \mathrm{l}$ nicotinamide (Gibco; Thermo Fisher Scientific, Inc.) and $1 \mathrm{~g} / 1$ insulin-transferrin-selenium (ITS; Sigma-Aldrich; Merck $\mathrm{KGaA}$ ) in stage 3 . All culture mediums were replaced every 2-3 days. The control group was cultured in medium without any supplementation. Cells were observed using an inverted light microscope every day.

Dithizone (DTZ) staining. Following induction, cells were washed with PBS, fixed with $4 \%$ paraformaldehyde at room temperature for $15 \mathrm{~min}$ and stained with DTZ (Sigma-Aldrich; Merck KGaA) staining solution for $30 \mathrm{~min}$ at room temperature. Stained cells were observed using a light microscope. The staining protocol was based on a study conducted by
Shiroi et al (18). Samples were assessed in triplicate. The assay was conducted in 60-mm dishes.

Immunofluorescence staining assays of IPCs markers. According to the aforementioned protocol, immunofluorescence staining was performed using primary antibodies targeted against the following: Insulin (1:200; cat. no. ab9823; Abcam), glucagon (1:200; cat. no. ab189279; Abcam), C-peptide (1:200; cat. no. ab82696; Abcam) and NK6 homeobox 1 (Nkx6.1; 1:200; cat. no. ab251565; Abcam). The nuclear was stained with DAPI (cat. no. C1002; Beyotime Institute of Biotechnology) for $20 \mathrm{~min}$ at room temperature. Samples were assessed in triplicate. The assay was conducted in 60-mm dishes.

Glucose-stimulated insulin secretion (GSIS). Following washing with PBS, differentiated IPCs were pre-cultured in low-glucose $(2 \mathrm{mM})$ buffer for $1 \mathrm{~h}$ to remove residual insulin. Cell clusters were washed twice with PBS and then incubated in low-glucose $(5 \mathrm{mM})$ buffer for $30 \mathrm{~min}$ at $37^{\circ} \mathrm{C}$. The supernatant was collected. Subsequently, cell clusters were washed twice in PBS and then incubated in high-glucose $(25 \mathrm{mM})$ buffer for $30 \mathrm{~min}$ at $37^{\circ} \mathrm{C}$. The supernatant was collected via centrifugation at $339 \mathrm{x}$ g for $10 \mathrm{~min}$ at room temperature. Cell clusters were dispersed into single cells using trypsin and the cell number was counted. Undifferentiated cell samples were used as controls. Supernatant samples containing secreted insulin were processed using the mouse insulin ELISA kit (cat. no. 0740; Pro Lab Marketing Pvt. Ltd.), which displays $0.019 \mathrm{ng} / \mathrm{ml}$ super sensitivity. The protocol was modified from the method described by Pagliuca et al (19) and Bai et al (20). Samples were assessed in triplicate. The assay was conducted in 60-mm dishes. The experiment was repeated three times.

Statistical analysis. Comparisons among multiple groups were analyzed using one-way ANOVA followed by the Tukey-Kramer post hoc test in GraphPad Prism 8 (GraphPad Software, Inc.). Comparisons between two groups were analyzed using an unpaired Student's t-test. Data are presented as the mean $\pm \mathrm{SD}$. $\mathrm{P}<0.05$ was considered to indicate a statistically significant difference.

\section{Results}

Morphological observation of PMSCs. Following culture for $12 \mathrm{~h}$, only a small number of cells were attached to the plate. Adherent cells gradually proliferated. In primary cultures, PMSCs were mixed with epithelial cells and other different types of cells. At passage 4, PMSCs were purified and 
A

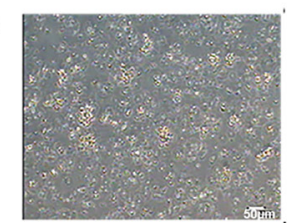

Culture for $\mathbf{O} \mathbf{h}$

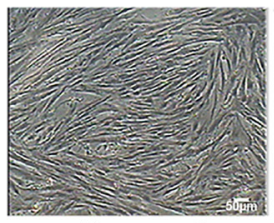

Passage 4

B O

C

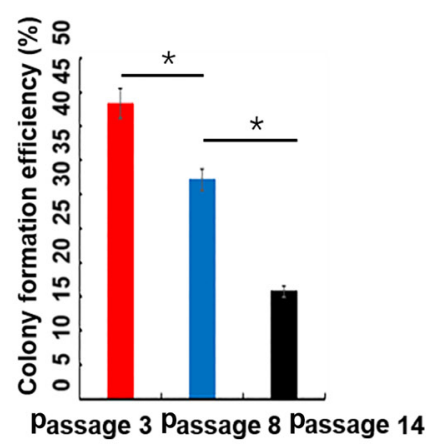

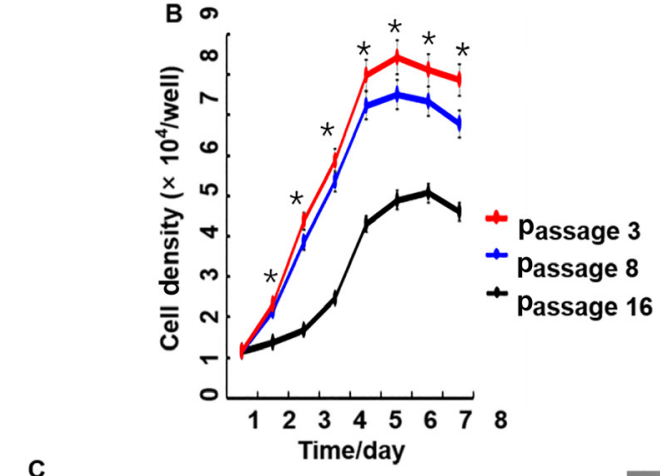

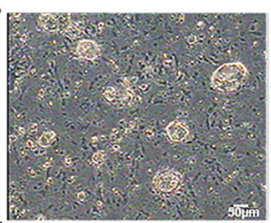

Culture for $12 \mathrm{~h}$

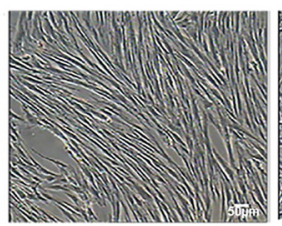

Passage 13

Passage 18

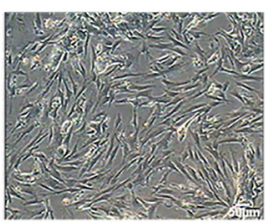

Passage 1

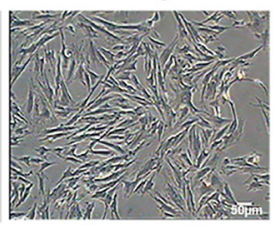

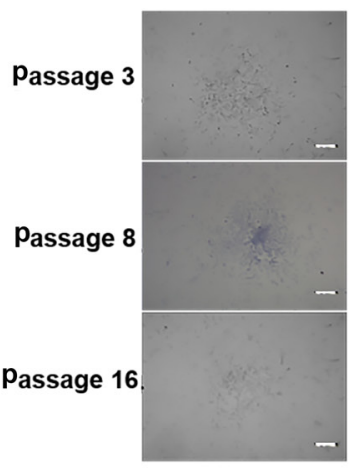

Figure 2. Morphology, growth curves and colony-forming efficiency of porcine PMSCs in vitro. (A) Morphology of cells at 0 and $12 \mathrm{~h}$, as well as passages 1, 4, 13 and 18; scale bar, $50 \mu \mathrm{m}$. (B) Growth curves of PMSCs. The growth curves of passage 3, 8 and 16 PMSCs were all sigmoidal. The growth rate of passage 16 cells was significantly slower than younger passage cells. The growth curves consisted of latent, logarithmic and plateau phases. The population doubling time, which was calculated from the growth curve, was $48 \mathrm{~h}$. Passage 3 has a significant difference with passage 16. (C) Colony-formation assay. Colony formation rates of PMSCs at passage 3, 8 and 14. Scale bar, $50 \mu \mathrm{m}$. ${ }^{*} \mathrm{P}<0.05$. PMSC, pancreas-derived mesenchymal stromal cell.

displayed fibroblast-like morphology, with long spindle-like and polygon-like cell features. At passage 18, PMSCs rapidly expanded and then cell proliferation slowed down, resulting in flattened cells with a lack of three-dimensional structure that displayed signs of senescence (Fig. 2A).

Growth kinetics. The growth curve of PMSCs displayed a typical ' $\mathrm{S}$ ' shape, cells underwent latency, logarithmic and plateau phases (Fig. 2B). However, passage 16 cells proliferated significantly slower compared with passage 3 and 8 cells. Based on the growth curve, the PDT of PMSCs was estimated to be $\sim 48 \mathrm{~h}$.

Colony-forming assay. At passage 3, 8 and 14, the colony-forming ability of PMSCs was detected by microscopy.

Colony-forming efficiencies were $43.57,32.48$ and $16.00 \%$ at passage 3, 8 and 14, respectively (Fig. 2C).

Immunofluorescence staining detection of PMSC surface antigens markers. Specific surface markers, including Vimentin, Nestin, Ngn3, Pdx1 and CD45, were detected by immunofluorescence staining in passage 4 cells (Fig. 3). The results demonstrated that PMSCs positively expressed Vimentin, Nestin, Ngn3 and Pdx1, but did not express CD45. Vimentin is an important marker of mesenchymal cells (17) and the results demonstrated that Vimentin was highly expressed in PMSCs.

Flow cytometry. The expression of cell surface antigens by PMSCs in early passage cells (up to passage 3 ) was analyzed by flow cytometry (Figs. 4 and 5). Cultured PMSCs were composed of a single phenotypic population. The positive rates of Vimentin, Pdx1, Ngn3, Nestin, CD73, CD90 and CD34 were 98.58, 96.23, 95.79, 37.12, 89.33, 91.53 and $1.07 \%$, respectively, which were calculated by 1 minus the rate of control rate.

Morphological alterations and DTZ staining of PMSC-derived IPCs. PMSCs at passage 3 were cultured in differentiation medium for 3 weeks. Induced cells were observed daily for morphological alterations. After 12 days, a number of small cell clusters formed and the number of cell clusters increased as cells differentiated (Fig. 6). After 3 weeks induction, cell clusters were stained with DTZ (Fig. 7) and showed a scarlet color.

Immunofluorescence staining assays of IPC markers. Differentiated cells were analyzed by performing an immunofluorescence staining assay (Fig. 8). The differentiated islet-like cell clusters expressed insulin and Nkx6.1, whereas the undifferentiated PMSCs around the cell clusters did not express insulin and Nkx6.1. Glucagon staining suggested that the differentiated cell clusters were monohormonal cells. IPCs were positive for insulin and C-peptide, which are stoichiometric byproducts of proinsulin processing (20). The results suggested that PMSCs were differentiated into IPCs using the optimized three-step culture procedure.

GSIS. Insulin secretion was evaluated by performing ELISAs. The results demonstrated that newly generated IPCs secreted insulin in response to glucose stimulation (Fig. 9). The differentiation rate of PMSCs was determined by flow cytometry. The results demonstrated that $79.82 \pm 1.36 \%$ of the total number of differentiated cells were insulin-positive (Fig. 10), which suggested that PMSCs had differentiated into functional IPCs.

\section{Discussion}

SCs have multiple differentiation potentials and display self-renewal capacities; under certain conditions, SCs significantly proliferate and differentiate into specific lineages (21). MSCs derived from the mesoderm can also differentiate into multiple mesodermal and nonmesodermal cell lineages in vitro $(21,22)$. Owing to their high proliferative ability, lack 


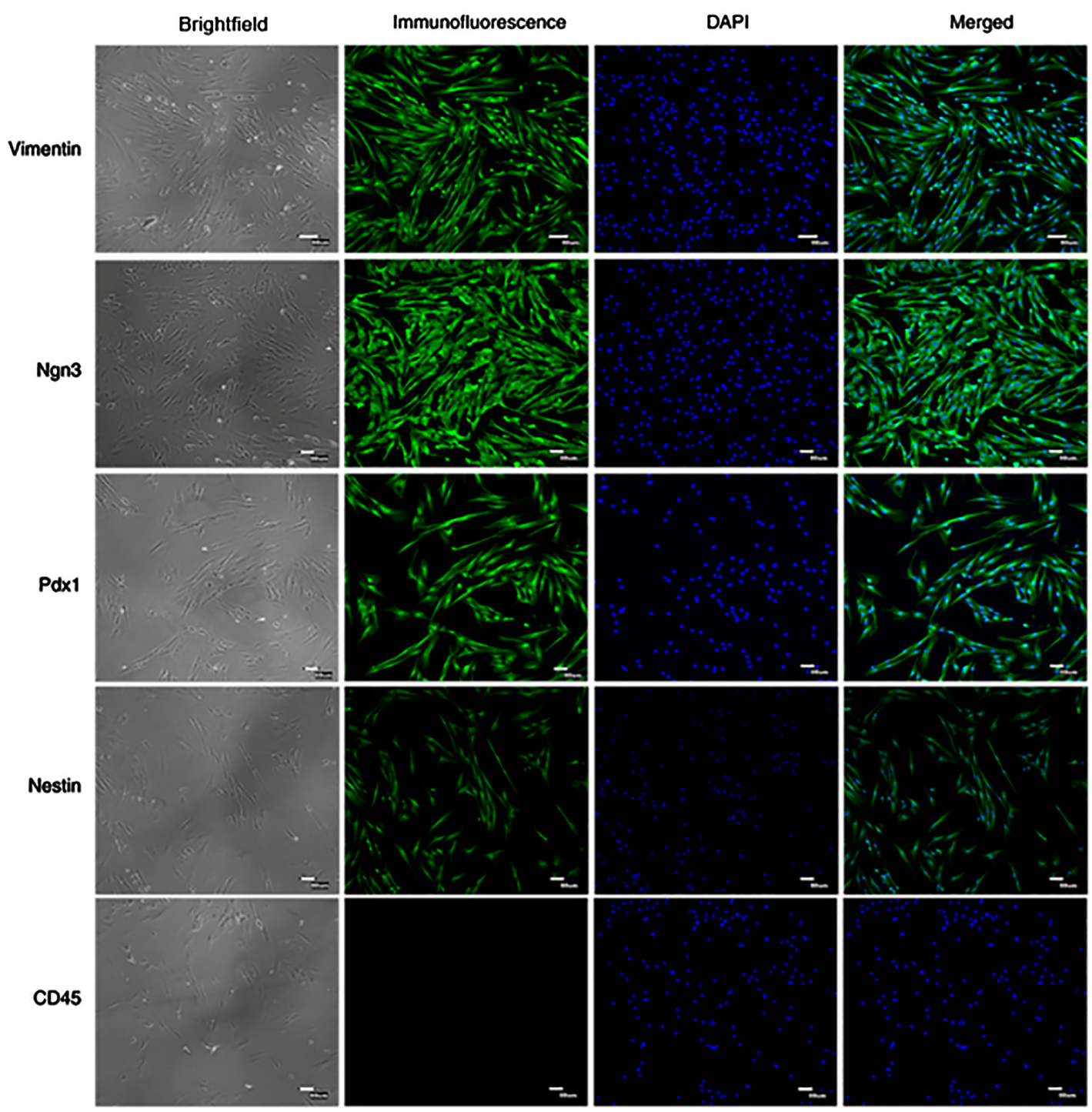

Figure 3. Immunofluorescence staining identification of PMSC specific markers. Expression of PMSC specific markers, including Vimentin, Ngn3, Pdx1, Nestin and CD45; scale bar, $80 \mu \mathrm{m}$. DAPI was used to stain the nuclei. Ngn3, neurogenin 3; Pdx1, pancreatic and duodenal homeobox 1; PMSC, pancreas-derived mesenchymal stromal cell.
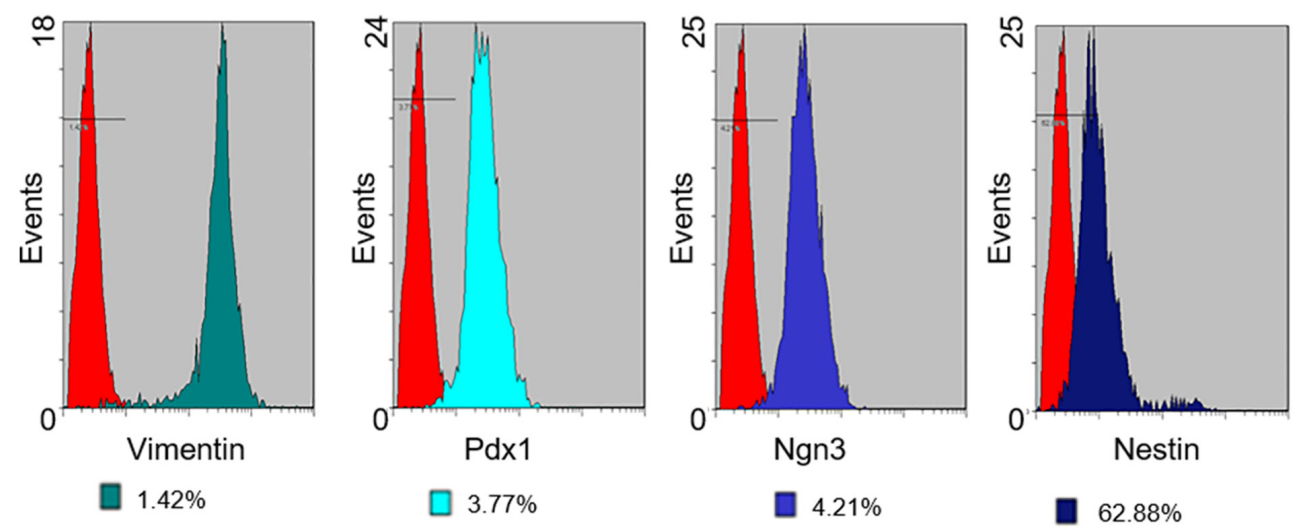

Figure 4. Flow cytometric analysis of PMSC expression of multiple antigens. PMSCs expressed Vimentin, Pdx1, Ngn3 and Nestin. The red histogram represents the control group. Ngn3, neurogenin 3; Pdx1, pancreatic and duodenal homeobox 1; PMSC, pancreas derived mesenchymal cell.

of adverse influence from allogeneic antigens and autoantigens, and teratoma formation abilities, MSCs are a promising source for clinical research (23). The differentiation of
MSCs into IPCs from bone marrow- (24-26), umbilical cord blood- $(27,28)$, adipose- $(29,30)$ and placenta-derived (31) MSCs has been described. 


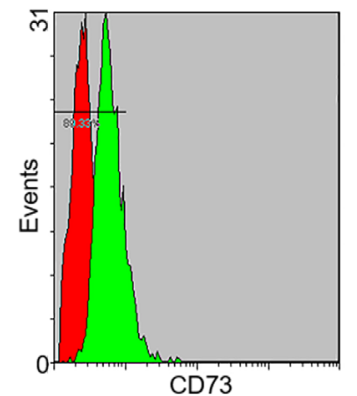

$89.33 \%$

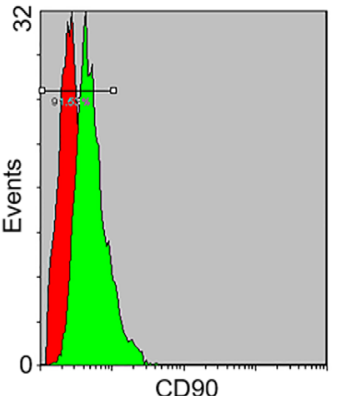

$91.53 \%$

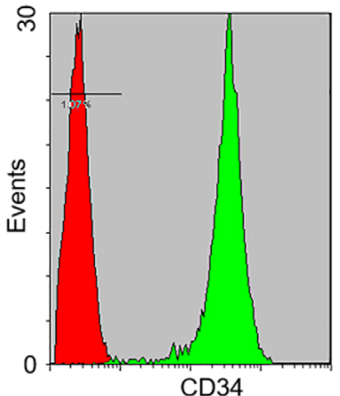

$1.07 \%$

Figure 5. Flow cytometric analysis of PMSC expression of mesenchymal cell antigens. PMSCs expressed CD73, CD90 and CD34. The red histogram represents the control group. PMSC, pancreas-derived mesenchymal stromal cell.
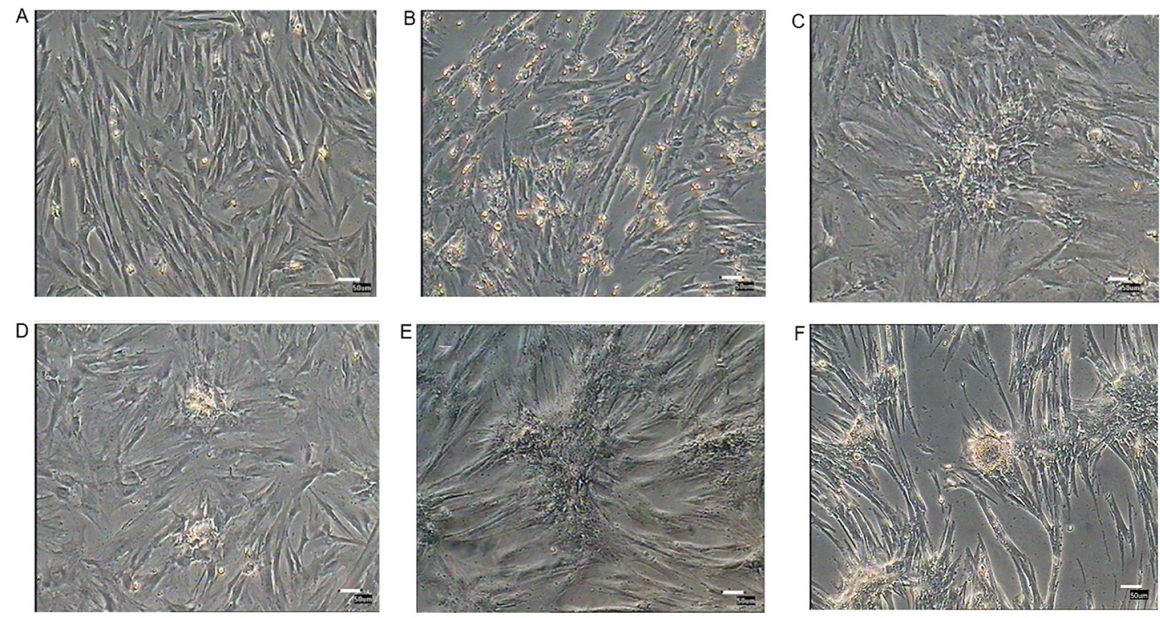

Figure 6. Morphological alterations of PMSCs during differentiation. (A) Cells prior to incubation with the differentiation medium. (B) During the stage one, PMSCs started to aggregate. (C) During stage one, cells clumped into small clusters. (D) During stage two, the cell cluster gradually increased. (E) During the stage two, cell clusters grew bigger and became more obvious. (F) During stage three, the cell cluster increased further. Scale bar, $50 \mu \mathrm{m}$. PMSC, pancreas-derived mesenchymal stromal cell.

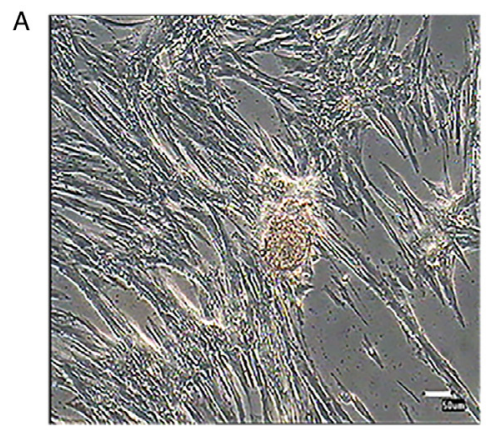

C

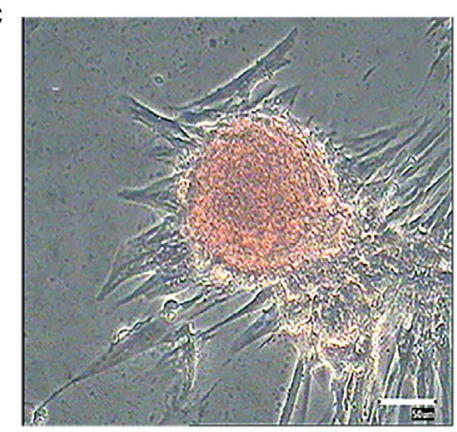

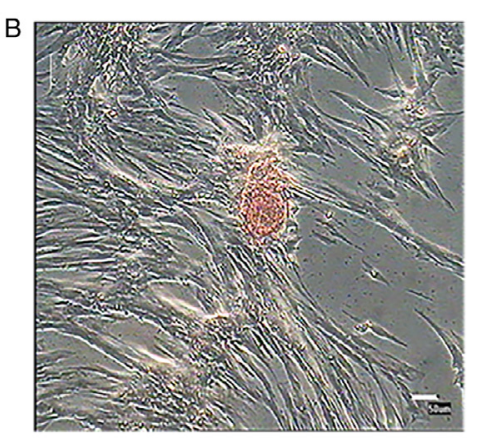

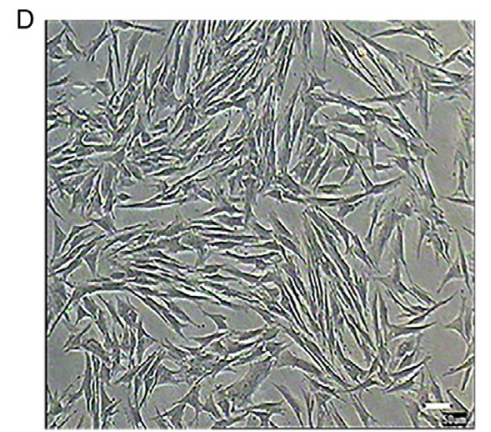

Figure 7. Morphological alterations of PMSCs identified by DTZ staining. Following induction for 21 days, PMSCs formed typical islet-like clusters, which were stained with DTZ. Insulin-producing cells were stained brown. (A) Before staining. (B) After staining (magnification, x40). (C) After staining (magnification, x100). (D) PMSCs without induction. Scale bar, $50 \mu \mathrm{m}$. DTZ, dithizone; PMSC, pancreas-derived mesenchymal stromal cell. 


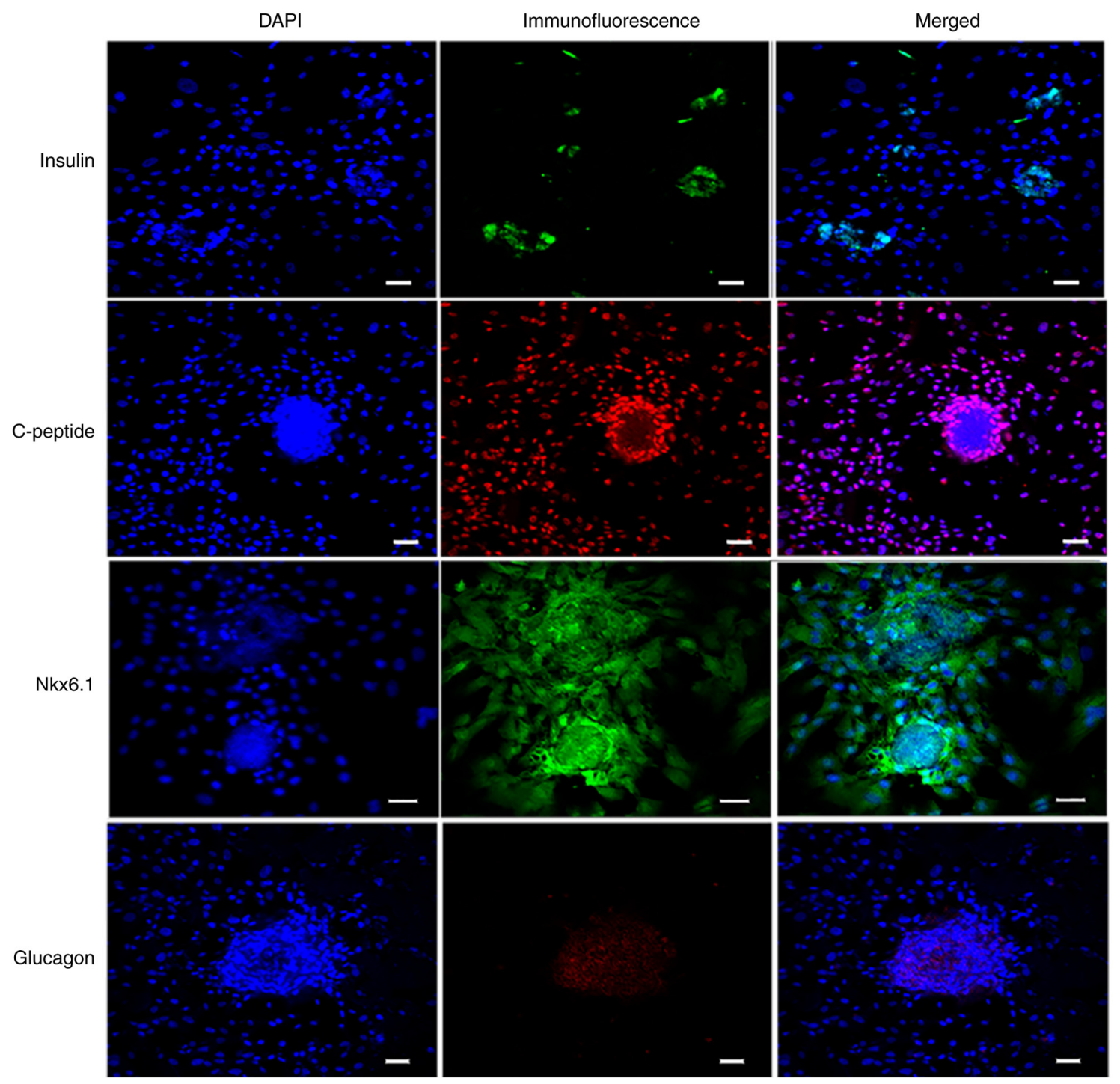

Figure 8. Identification of IPCs by immunocytochemistry. IPC expression of islet $\beta$ cell specific markers, including insulin, C-peptide, glucagon and Nkx6.1; cell nuclei were stained with DAPI. Scale bar, $50 \mu \mathrm{m}$. IPC, insulin-producing cell; Nkx6.1, NK6 homeobox 1.

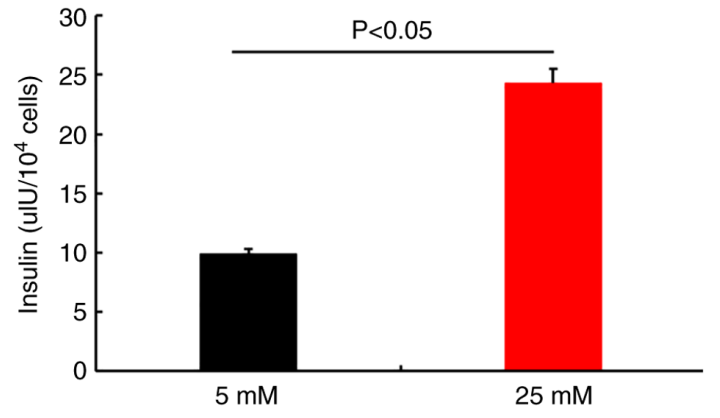

Figure 9. IPC GSIS indicates physiological functions in vitro. IPCs were treated with either 5 or $25 \mathrm{mM}$ glucose for $30 \mathrm{~min}$. Subsequently, insulin secretion was measured by ELISA. GSIS, glucose-stimulated insulin secretion; IPC, insulin-producing cell.

The present study described an optimized three-step induction protocol, which was used to obtain IPCs from porcine PMSCs. Increasing evidence has demonstrated that SCs present in pancreatic ducts, islets and acini display a specific potential for differentiation (13). Compared with the two-step induction protocol (13), the clear morphology of islet-like cells was a notable benefit observed with the three-step protocol used in the present study. In addition, pancreatic SCs are derived from pancreas ductal tissues and display a cobblestone like morphology, whereas PMSCs are derived from acinar tissue and display a fibroblast-like morphology (20). PMSCs can be isolated using a simpler protocol and display a higher proliferation ability than pancreas stem cells (21). The present study induced PMSC transformation into IPCs using a three-step protocol. Firstly, cell growth in a favorable microenvironment was sustained by adding bFGF and EGF, which promoted cell development. Secondly, Activin A, B27 KGF and nicotinamide were used to stimulate PMSC neural and entoderm differentiation. Finally, ITS, Activin A, nicotinamide and exendin-4 were added to the culture medium to sustain entoderm differentiation and to stimulate cells into an islet-like morphology. In addition, compared with other adult SCs, PMSCs are more likely to form IPCs (21), which suggested that they may serve as an ideal candidate for diabetes therapy.

The present study demonstrated the successful expansion of porcine PMSCs in vitro and the differentiation into 

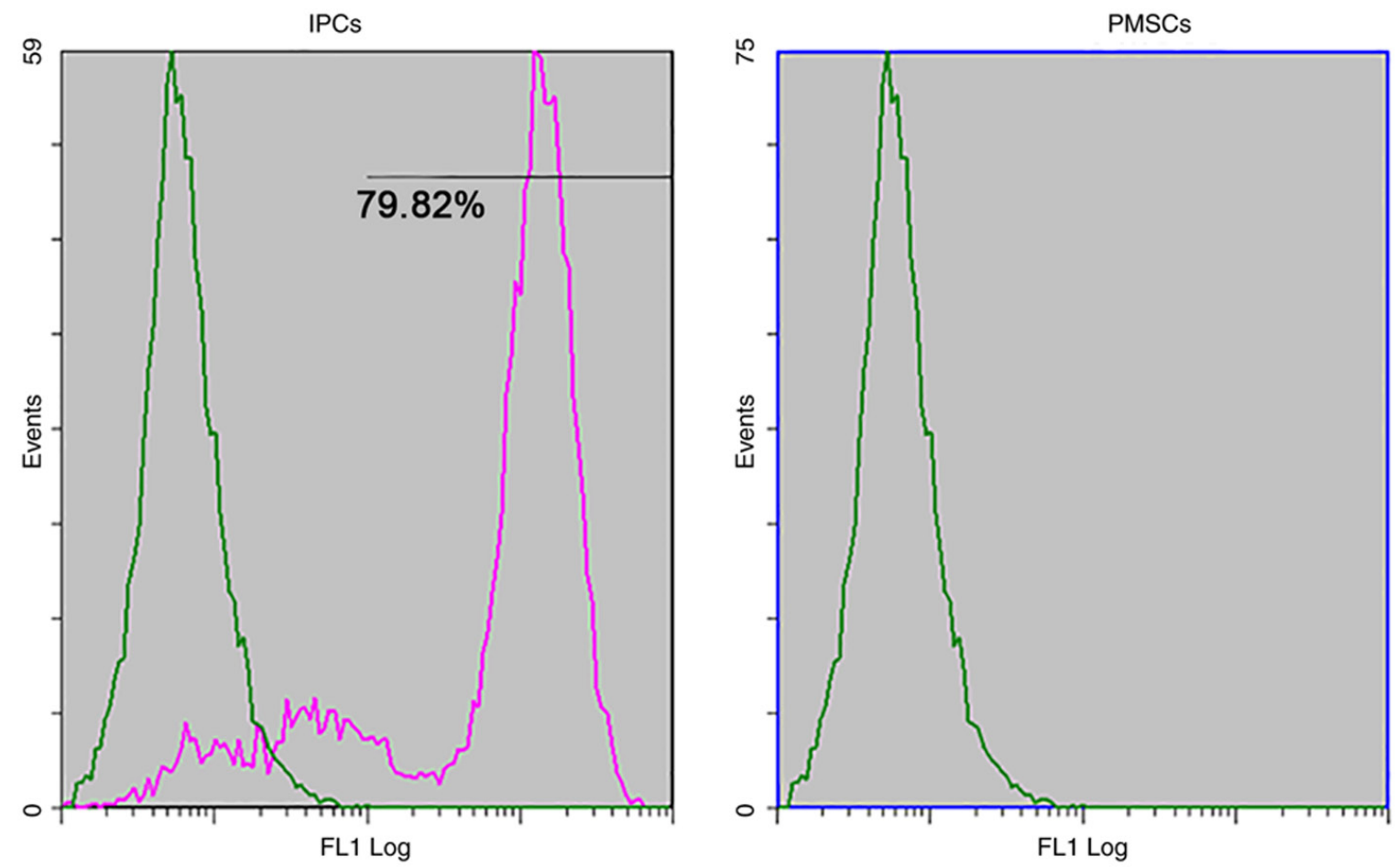

Figure 10. Flow cytometric analysis of insulin-positive cells. Among the differentiated IPCs, $79.82 \%$ were insulin positive. The green histogram represents the control group. IPC, insulin-producing cell; PMSC, pancreas-derived mesenchymal stromal cell.

IPC clusters after 3 weeks induction; PMSCs aggregated and formed islet-like cell clusters that were DTZ-positive and expressed specific markers of $\beta$ cells. The GSIS experiment results indicated that the differentiated cells secreted insulin in response to glucose alterations. The weak expression of insulin indicated that the protocol requires further revision. Thus, the function of induced IPCs with the three-step protocol requires additional investigation in vivo using animal models and other MSC types, such as bone marrow, adipose and other cells in vitro.

The results of the present study indicated that PMSCs displayed a strong self-renewal capacity and expressed the surface markers of MSCs. The present study also provided evidence for the generation of functional IPCs from PMSCs using an optimized three-step protocol. However, the present study only investigated the function of IPCs in vitro and lacked investigation using an in vivo animal model. In addition, the three-step protocol required multiple factors and lasted for 3 weeks; thus, attempts should be made to simplify and optimize the protocol further. The application of the three-step protocol in other cells types, such as MSCs, should also be investigated in the future as it may be of great significance for the treatment of diabetes.

\section{Acknowledgements}

Not applicable.

\section{Funding}

The present study was supported by The National Science Foundation of China (grant nos. 31201765, 31272403 and
31472064) and the earmarked fund for Modern Agri-industry Technology Research System (grant no. nycytx-40-01).

\section{Availability of data and materials}

The datasets used and/or analyzed during the current study are available from the corresponding author on reasonable request.

\section{Authors' contributions}

SZ and QW performed the experiments, contributed to data analysis, drafted the manuscript and were responsible for the authenticity of data. HJ and HL revised the draft manuscript in the Results and Discussion sections and also acquire the raw data. QY and JY performed the data analysis. WG provided the final version publication of the manuscript, designed the project and also interpretated the raw data. All authors read and approved the final manuscript.

\section{Ethics approval and consent to participate}

All animal procedures were approved by the Institutional Animal Care and Use Committee of Chinese Academy of Agricultural Sciences (approval no. IAS 2018-19).

\section{Patient consent for publication}

Not applicable.

\section{Competing interests}

The authors declare that they have no competing interests. 


\section{References}

1. Guariguata L, Whiting DR, Hambleton I, Beagley J, Linnenkamp U and Shaw JE: Global estimates of diabetes prevalence for 2013 and projections for 2035. Diabetes Res Clin Pract 103: 137-149, 2014.

2. De Paoli M and Werstuck GH: Role of estrogen in type 1 and type 2 diabetes mellitus: A review of clinical and preclinical data. Can J Diabetes 44: 448-452, 2020.

3. Zhou Q and Melton DA: Pancreas regeneration. Nature 557: 351-358, 2018.

4. Pan FC and Brissova M: Pancreas development in humans. Curr Opin Endocrinol Diabetes Obes 21: 77-82, 2014.

5. Bakhti M, Böttcher A and Lickert $\mathrm{H}$ : Modelling the endocrine pancreas in health and disease. Nat Rev Endocrinol 15: 155-171, 2019.

6. Fathi E, Farahzadi R and Sheikhzadeh N: Immunophenotypic characterization, multi-lineage differentiation and aging of zebrafish heart and liver tissue-derived mesenchymal stem cells as a novel approach in stem cell-based therapy. Tissue Cell 57: $15-21,2019$.

7. Arnold K, Sarkar A, Yram MA, Polo JM, Bronson R, Sengupta S, Seandel M, Geijsen N and Hochedlinger K: Sox2(+) adult stem and progenitor cells are important for tissue regeneration and survival of mice. Cell Stem Cell 9: 317-329, 2011.

8. Gross JB and Hanken J: Use of fluorescent dextran conjugates as a long-term marker of osteogenic neural crest in frogs. Dev Dyn 230: 100-106, 2004.

9. Yu F, Wei R, Yang J, Liu J, Yang K, Wang H, Mu Y and Hong T: FoxO1 inhibition promotes differentiation of human embryonic stem cells into insulin producing cells. Exp Cell Res 362: 227-234, 2018.

10. Tan J, Liu L, Li B, Xie Q, Sun J, Pu H and Zhang L: Pancreatic stem cells differentiate into insulin-secreting cells on fibroblast-modified PLGA membranes. Mater Sci Eng C Mater Biol Appl 97: 593-601, 2019.

11. Daryabor G, Shiri EH and Kamali-Sarvestani E: A simple method for the generation of insulin producing cells from bone marrow mesenchymal stem cells. In Vitro Cell Dev Biol Anim 55: 462-471, 2019.

12. Cooper TT, Sherman SE, Bell GI, Ma J, Kuljanin M, Jose SE, Lajoie GA and Hess DA: Characterization of a Vimentin high/Nesti ${ }^{\text {nhigh }}$ proteome and tissue regenerative secretome generated by human pancreas-derived mesenchymal stromal cells. Stem Cells 38: 666-682, 2020.

13. Lee S, Moon S, Oh JY, Seo EH, Kim YH, Jun E, Shim IK and Kim SC: Enhanced insulin production and reprogramming efficiency of mesenchymal stem cells derived from porcine pancreas using suitable induction medium. Xenotransplantation 26: e12451, 2019

14. Iqbal MA, Hong K, Kim JH and Choi Y: Severe combined immunodeficiency pig as an emerging animal model for human diseases and regenerative medicines. BMB Rep 52: 625-634, 2019.

15. Fu X, Liu G, Halim A, Ju Y, Luo Q and Song AG: Mesenchymal stem cell migration and tissue repair. Cells 8: 784, 2019

16. Xu J, Yu L, Guo J, Xiang J, Zheng Z, Gao D, Shi B, Hao H, Jiao D, Zhong L, et al: Generation of pig induced pluripotent stem cells using an extended pluripotent stem cell culture system. Stem Cell Res Ther 10: 193, 2019.

17. Kleeblatt J, Schubert JK and Zimmermann R: Detection of gaseous compounds by needle trap sampling and direct thermal-desorption photoionization mass spectrometry: Concept and demonstrative application to breath gas analysis. Anal Chem 87: 1773-1781, 2015.
18. Shiroi A, Yoshikawa M, Yokota H, Fukui H, Ishizaka S, Tatsumi K and Takahashi Y: Identification of insulin-producing cells derived from embryonic stem cells by zinc-chelating dithizone. Stem Cells 20: 284-292, 2002.

19. Pagliuca FW, Millman JR, Gürtler M, Segel M, Van Dervort A, Ryu JH, Peterson QP, Greiner D and Melton DA: Generation of functional human pancreatic $\beta$ cells in vitro. Cell 159: 428-439, 2014.

20. Bai C, Gao Y,Zhang X, Yang W and Guan W: MicroRNA-34c acts as a bidirectional switch in the maturation of insulin-producing cells derived from mesenchymal stem cells. Oncotarget 8: 106844-106857, 2017

21. Liu M and Han ZC: Mesenchymal stem cells: biology and clinical potential in type 1 diabetes therapy. J Cell Mol Med 12: $1155-1168,2008$

22. Zhang S, Zhu Z, Wang Y, Liu S, Zhao C, Guan W and Zhao Y: Therapeutic potential of Bama miniature pig adipose stem cells induced hepatocytes in a mouse model with acute liver failure. Cytotechnology 70: 1131-1141, 2018.

23. Venkatesh K and Sen D: Mesenchymal stem cells as a source of dopaminergic neurons: A potential cell based therapy for parkinson's disease. Curr Stem Cell Res Ther 12: 326-347, 2017.

24. Gabr MM, Zakaria MM, Refaie AF, Khater SM, Ashamallah SA, Ismail AM, El-Halawani SM and Ghoneim MA: Differentiation of human bone marrow-derived mesenchymal stem cells into insulin-producing cells: Evidence for further maturation in vivo. Biomed Res Int 2015: 575837, 2015.

25. Li X, Huang H, Liu X, Xia H and Li M: In vitro generation of insulin-producing cells from the neonatal rat bone marrow mesenchymal stem cells. Xi Bao Yu Fen Zi Mian Yi Xue Za Zhi 31: 346-349, 2015 (In Chinese).

26. Zhang S, Zhao C, Liu S, Wang Y, Zhao Y, Guan W and Zhu Z: Characteristics and multi-lineage differentiation of bone marrow mesenchymal stem cells derived from the Tibetan mastiff. Mol Med Rep 18: 2097-2109, 2018.

27. Van Pham P, Thi-My Nguyen P, Thai-Quynh Nguyen A, Minh Pham V, Nguyen-Tu Bui A, Thi-Tung Dang L, Gia Nguyen K and Kim Phan N: Improved differentiation of umbilical cord blood-derived mesenchymal stem cells into insulin-producing cells by PDX-1 mRNA transfection. Differentiation 87: 200-208, 2014.

28. Tsai PJ, Wang HS, Shyr YM, Weng ZC, Tai LC, Shyu JF and Chen TH: Transplantation of insulin-producing cells from umbilical cord mesenchymal stem cells for the treatment of streptozotocin-induced diabetic rats. J Biomed Sci 19: 47, 2012.

29. Zhou H, Yang J, Xin T, Li D, Guo J, Hu S, Zhou S, Zhang T, Zhang Y, Han T and Chen Y: Exendin-4 protects adipose-derived mesenchymal stem cells from apoptosis induced by hydrogen peroxide through the PI3K/Akt-Sfrp2 pathways. Free Radic Biol Med 77: 363-375, 2014.

30. Karaoz E, Okcu A, Ünal ZS, Subasi C, Saglam O and Duruksu G: Adipose tissue-derived mesenchymal stromal cells efficiently differentiate into insulin-producing cells in pancreatic islet microenvironment both in vitro and in vivo. Cytotherapy 15: 557-570, 2013.

31. Kadam S, Muthyala S, Nair P and Bhonde R: Human placenta-derived mesenchymal stem cells and islet-like cell clusters generated from these cells as a novel source for stem cell therapy in diabetes. Rev Diabet Stud 7: 168-182, 2010.

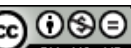

This work is licensed under a Creative Commons Attribution-NonCommercial-NoDerivatives 4.0 International (CC BY-NC-ND 4.0) License. 